\title{
Sonographic Evaluation of Endometrial Thickness in Postmenopausal Women- A Systemic Review
}

\author{
Sybil Rose* \\ Lecturer, Superior College Lahore, University Campus, 17-KM Raiwind Road, Kot Arain, Lahore, Pakistan \\ Syeda Khadija \\ Lecturer, The University of Lahore, Lahore Campus, 1-KM Defence Road, Lahore, Pakistan \\ Rana Muhammad Athar Azeem Shams \\ Lecturer, Superior College Lahore, University Campus, 17-KM Raiwind Road, Kot Arain, Lahore, Pakistan
}

Ambreen Sadaf

Lecturer, The University of Lahore, Lahore Campus, 1-KM Defence Road, Lahore, Pakistan

Maria Shahzadi

Lecturer, The University of Lahore, Lahore Campus, 1-KM Defence Road, Lahore, Pakistan

Azmat Tahira

Lecturer, The University of Lahore, Lahore Campus, 1-KM Defence Road, Lahore, Pakistan

\begin{abstract}
The research is financed by Asian Development Bank. No. 2006-A171(Sponsoring information)
Abstract

Postmenopausal bleeding (PMB) is highly suspicious of being a sign for the presence of endometrial cancer (EC) and around $5-12 \%$ of PMB results from EC. Endometrial cancer is the most common gynecologic malignancy in developed countries. A thin endometrium $(\leq 4 \mathrm{~mm})$ has a very high negative predictive value $(99 \%)$. A thickened endometrium has been regarded as an indication for an invasive evaluation. Endometrial sampling is recommended, with a cutoff value of $4 \mathrm{~mm}$ or $5 \mathrm{~mm}$, for symptomatic postmenopausal women. However, different guidelines use different cut-off values of endometrial thickness, varying from $3 \mathrm{~mm}-5 \mathrm{~mm}$, for excluding endometrial malignancy. The widespread use of sonography has also allowed the incidental finding of endometrial thickening in asymptomatic postmenopausal women. There are few data on the workup of these women, and the best cut-off value for the endometrial thickness that should warrant endometrial sampling is not known. However, it has been suggested that the 4 - or 5 - mm limits generally used for excluding malignancy in symptomatic postmenopausal women are not transferable to asymptomatic patients. The objective of this study was to sonographic evaluation of endometrial thickness in postmenopausal women. About 9 studies were selected for this systemic review. We extracted the following information: Study design, Age of patient mean and range, Duration of menopause mean and range, Body mass index mean and range, and Endometrial thickness mean and range. From the above analysis we concluded that, the endometrial thickness with a recommended change in the cut-off to $3 \mathrm{~mm}$ in routine ultrasound practice should be done in high risk women to detect malignancy earlier in postmenopausal women and ultrasound is proved to be a useful tool for the disgnostic purpose.
\end{abstract}

Keywords: Postmenopausal bleeding, Endometrial thickness, Endometrial Carcinoma.

DOI: $10.7176 / \mathrm{JHMN} / 82-04$

Publication date: November $30^{\text {th }} 2020$

\section{Introduction}

Postmenopausal bleeding (PMB) is a frequent event in postmenopausal women and represents up to $10 \%$ of all visits in private gynecological practice. The incidence of PMB in all postmenopausal women is approximately about $10 \%$, mostly caused by benign findings such as endometrial hyperplasia or atrophy or benign polyps ${ }^{(1,2)}$. However, PMB is also highly suspicious of being a sign for the presence of endometrial cancer (EC) and around $5-12 \%$ of $\mathrm{PMB}$ results from $\mathrm{EC}^{(3)}$.

\subsection{Endometrial Cancer}

Endometrial cancer is the most common gynecologic malignancy in developed countries, and endometrial cancer is diagnosed in more than $90 \%$ of women with postmenopausal vaginal bleeding ${ }^{(4)}$. For such postmenopausal women, the risk of endometrial carcinoma ranges from $4.9 \%$ to $11.5 \%$ and therefore, early and accurate diagnosis is important and postmenopausal bleeding should be properly investigated ${ }^{(5)}$. A thin endometrium $(\leq 4 \mathrm{~mm})$ has a very high negative predictive value $(99 \%)$ and histological sampling may be $\operatorname{avoided}^{(6)}$. 


\subsection{Diagnosis}

In the past, the gold standard for clinical investigation of postmenopausal bleeding was institution - based dilatation and curettage (D\&C), but now there are a number of office - based methods, such as endometrial biopsy and hysteroscopy, for the evaluation of women with this complaint and endometrial biopsy has almost completely replaced D\&C. Outpatient endometrial biopsy performed with a Pipelle sampling device (Cooper Surgical, Trumbull, CT) is simple to perform, relatively inexpensive, and highly sensitive in detecting endometrial carcinoma $^{(7,8)}$.

In the present, sonographic measurement of endometrial thickness has also become increasingly popular as a non-invasive diagnostic tool for assessing uterine pathology in patients with postmenopausal bleeding. A thickened endometrium has been regarded as an indication for an invasive evaluation. Endometrial sampling is recommended, with a cutoff value of $4 \mathrm{~mm}$ or $5 \mathrm{~mm}$, for symptomatic postmenopausal women. However, different guidelines use different cut-off values of endometrial thickness, varying from $3 \mathrm{~mm}-5 \mathrm{~mm}$, for excluding endometrial malignancy ${ }^{(9-12)}$.

The widespread use of sonography has also allowed the incidental finding of endometrial thickening in asymptomatic postmenopausal women. There are few data on the workup of these women, and the best cut-off value for the endometrial thickness that should warrant endometrial sampling is not known. However, it has been suggested that the 4 - or 5 - mm limits generally used for excluding malignancy in symptomatic postmenopausal women are not transferable to asymptomatic patients ${ }^{(13,14)}$.

Therefore, our aim in this study was to assess the diagnostic value of sonographically, measured endometrial thickness in predicting endometrial pathologies in postmenopausal women with vaginal bleeding and in those with asymptomatic thickened endometrium ( $\geq 5 \mathrm{~mm}$ ). To accomplish this, we investigated the best cutoff values to use for discriminating between postmenopausal women with intrauterine pathologies and those without such pathology.

\section{Materials and Methods}

2.1 Data sources and searches

One single investigator searched in two databases (PubMed and Web of Science) for studies reporting on endometrial thickness in postmenopausal women, published between January 2014 and November 2020. Language limit was set to English.

The terms used for the search included "endometrial thickness", "postmenopausal," and "postmenopause." For example, for search in PubMed, we used the following Medical Subject Heading (MeSH) terms: (endometrial [All Fields] AND thickness [All Fields] AND asymptomatic [All Fields] AND ("postmenopause" [MeSH Terms] OR “postmenopause" [All Fields]) AND postmenopausal [All Fields]).

\subsection{Study selection}

One investigator screened the titles and abstracts identified by the search for excluding duplicate and irrelevant articles (studies not dealing with the topic, reviews, and letter - to - editors). We also excluded studies with less than 150 women included (this was an arbitrary criterion, just aiming for obtaining more robust data), studies including premenopausal and/or perimenopausal women, studies including women taking tamoxifen (TMX), hormonal replacement therapy (HRT), aromatase inhibitors, or any other selective estrogen receptor modulator (SERM) were excluded.

Then, full text of the remaining articles was independently reviewed by three investigators for identifying potential eligible studies based on the following criteria: studies reporting on ET in postmenopausal women.

\subsection{Data extraction}

One investigator independently extracted data for each study ultimately included. We extracted the following information: Study design, Age of patient mean and range, Duration of menopause mean and range, Body mass index mean and range, and Endometrial thickness mean and range.

\section{Discussion}

In 2014 Bahadir Saatli et, al., did a retrospective study in 530 patients on the role of endometrial thickness for detecting endometrial pathologies in asymptomatic postmenopausal women, in which he evaluated the significance of endometrial sampling in asymptomatic, bleeding-free postmenopausal women who have endometrial thickness greater or equal to $5 \mathrm{~mm}$. A transvaginal ultrasonography was performed. About five hundered and thirty asymptomatic postmenopausal women underwent ultrasonographic evaluation with subsequent endometrial sampling. The mean age was 61 (range: 46-82). The mean duration of menopause 16 (range: 1-48). The mean body mean index (BMI) 27.4 (range: 18-48). The mean endometrial stripe thickness was $8.7 \mathrm{~mm}$ (range: 6-26). They concluded that, 106 investigations were performed to detect one case of adenocarcinoma. Although this is a high number per case detection of endometrial adenocarcinoma, considering 
the rising incidence of endometrial cancer, large prospective trials with surrogate criteria for asymptomatic women are needed to investigate the importance of thickened endometrial stripe in postmenopausal women ${ }^{(15)}$.

In 2015 AS-W Wong et. al., did a retrospective study in 4383 women with post-menopausal bleeding between 2002 and 2013, on reappraisal of endometrial thickness for the detection of endometrial cancer in postmenopausal bleeding using transvaginal ultrasonic measurement of ET and endometrial biopsies were obtained in women presenting with PMB, endometrial histology was used as the reference standard to calculate accuracy estimates. In $3.8 \%$ of women endometrial cancer was diagnosed. The mean age is 55 (range: 52-62). The mean duration of menopause 50 (range: 48-52). The mean body of index 23.9 (range: 21.6-26.6). The mean endometrial thickness 15.7 (range: 9.5-23.0). They concluded that, Transvaginal ultrasound using a 3-mm cut-off has high sensitivity for detecting endometrial cancer and can identify women with PMB who are highly unlikely to have endometrial cancer, thereby avoiding more invasive endometrial biopsy ${ }^{(16)}$.

In 2017 Amelie Schramm et. al., did a retrospective study in 254 patients on value of endometrial thickness assessed by transvaginal ultrasound for the prediction of endometrial cancer in patients in with postmenopausal bleeding. The objective of his study was to asses the diagnostic validity of transvaginal ultrasound (TVUS) measurements of endometrial thickness (ET) in patients with postmenopausal bleeding for the detection of endometrial carcinoma. The mean age 64 (range: 40-92). The mean body mass index 28 (16.9-103.6). The mean endometrial thickness $>4 \mathrm{~mm}(83.1 \%) ;>10 \mathrm{~mm}(49.9 \%)$ and $>15 \mathrm{~mm}(28.6 \%)$. They concluded that, routine use of endometrial thickness measurement with TVUS does not seem to be an effective diagnostic tool for endometrial cancer because it has a low diagnostic performance in asymptomatic postmenopausal women. Further prospective studies are required to assess the endometrial thickness measurement with TVUS as a screening method in these women $^{(1)}$.

In 2018 Alper Basbug et.al., did a retrospective study in 155 patients showing the relationship between adnexal mass and endometrial thickness in post-menopausal women. The aim was to employ transvaginal ultrasonography in the evaluation of adnexal masses synchronously seen in postmenopausal women with increased endometrial thickness. The standard deviation age $57.75 \pm 11.89$. The standard deviation duration of menopuase 11.23 \pm 7.65. The standard deviation body mass index 33.60 \pm 3.90 . The standard deviation endometrial thickness $11.73 \pm 5.97$. They concluded that, in postmenopausal women, adnexal masses can be seen simultaneously with increased endometrial thickness. In this regard, transvaginal ultrasound offers important opportunities for evaluation of both the endometrium and adnexa. Pre-surgery transvaginal ultrasound as well as multivariate serum markers may be used in evaluation models ${ }^{(17)}$.

In 2019 Magdalena Pirog et.al., did a retrospective study in 57 patients on the thin red line-post menopausal abnormal uterine bleeding with endometrial thickness less that $4 \mathrm{~mm}$. The aim of his study was to evaluate the risk of endometrial malignancy in postmenopausal women with abnormal uterine bleeding (AUB) with endometrial thickness $\leq 4 \mathrm{~mm}$. The standard deviation age 56.75 \pm 6.6 . The standard deviation duration of menopuase 51.1 \pm 4.3 . The mean body mass index 25.3 (range: 23.5-29.4). The standard deviation endometrial thickness 3.2 \pm 0.6 . They concluded that, Postmenopausal women with abnormal uterine bleed and endometrial echo $\leq 4 \mathrm{~mm}$ are less likely to have a malignant condition. However, the role of histological evaluation cannot be undermined, especially in women at high risk of endometrial cancer, and routine endometrial biopsy should be considered. We recommend a change in the cut-off to $2 \mathrm{~mm}$ in routine practice ${ }^{(18)}$.

In 2020 Dinesh Palipana et.al., did a retrospective study in 222 patients invsetigating women with postmenopausal bleeding. The mean age 60.9 (range: 59.7-62.2). The mean duration of menopuase 31.9. The range body mass index 30.6-33.1. The range endometrial thickness $>4 \mathrm{~mm}$ (7.4-9.1) and $>10 \mathrm{~mm}(10.9-17.6)$. They concluded that, a limit of $3 \mathrm{~mm}$ for endometrial thickness ET in postmenopausal bleeding PMB, along with office endometrial biopsy, should be considered to ensure timely diagnoses ${ }^{(19)}$.

\section{Conclusion}

From the above analysis we concluded that, the endometrial thickness with a recommended change in the cut-off to $3 \mathrm{~mm}$ in routine ultrasound practice should be done in high risk women to detect malignancy earlier in postmenopausal women and ultrasound is proved to be a useful tool for the disgnostic purpose.

\section{References}

Schramm A, Ebner F, Bauer E, Janni W, Friebe-Hoffmann U, Pellegrino M, et al. Value of endometrial thickness assessed by transvaginal ultrasound for the prediction of endometrial cancer in patients with postmenopausal bleeding. Archives of Gynecology and Obstetrics. 2017;296(2):319-26.

Carugno J. Clinical management of vaginal bleeding in postmenopausal women. Climacteric. 2020:1-7.

Dueholm M, Hjorth I, Dahl K, Pedersen L, Ørtoft G. Identification of endometrial cancers and atypical hyperplasia: development and validation of a simplified system for ultrasound scoring of endometrial pattern. Maturitas. 2019;123:15-24.

Saso S, Chatterjee J, Georgiou E, Ditri AM, Smith JR, Ghaem-Maghami S. Endometrial cancer. Bmj. 2011 ;343. 
Seckin B, Cicek MN, Dikmen AU, Bostancı EI, Muftuoglu KH. Diagnostic value of sonography for detecting endometrial pathologies in postmenopausal women with and without bleeding. Journal of Clinical Ultrasound. 2016;44(6):339-46.

Fakhar S, Saeed G, Khan AH, Alam AY. Validity of pipelle endometrial sampling in patients with abnormal uterine bleeding. Annals of Saudi medicine. 2008;28(3):188-91.

Abdelazim IA, Abdelrazak KM, Elbiaa AA, Al-Kadi M, Yehia AH. Accuracy of endometrial sampling compared to conventional dilatation and curettage in women with abnormal uterine bleeding. Archives of gynecology and obstetrics. 2015;291(5):1121-6.

Malanowska-Stega Z, Delpriore G. Simultaneous multiple method out-patient uterus biopsy device and method. Google Patents; 2020.

Yasa C, Dural O, Bastu E, Ugurlucan FG, Nehir A, İyibozkurt AC. Evaluation of the diagnostic role of transvaginal ultrasound measurements of endometrial thickness to detect endometrial malignancy in asymptomatic postmenopausal women. Archives of gynecology and obstetrics. 2016;294(2):311-6.

Van Hanegem N, Breijer M, Khan K, Clark T, Burger M, Mol B, et al. Diagnostic evaluation of the endometrium in postmenopausal bleeding: an evidence-based approach. Maturitas. 2011;68(2):155-64.

Patel V, Wilkinson EJ, Chamala S, Lu X, Castagno J, Rush D. Endometrial thickness as measured by transvaginal ultrasound and the corresponding histopathologic diagnosis in women with postmenopausal bleeding. International Journal of Gynecological Pathology. 2017;36(4):348-55.

Wolfman W, Leyland N, Heywood M, Singh SS, Rittenberg DA, Soucy R, et al. Asymptomatic endometrial thickening. Journal of Obstetrics and Gynaecology Canada. 2010;32(10):990-9.

Carolin Solomi V. A Prospective Observational Study to Determine the Aetiology of Postmenopausal Bleeding and Correlation of Endometrial Thickness in Endometrial Carcinoma in Our Population: Christian Medical College, Vellore; 2017.

Liberis V, Tsikouras P, Christos Z, Ammari A, Dislian V, Koutlaki N, et al. The contribution of hysteroscopy to the detection malignancy in symptomatic postmenopausal women. Minimally Invasive Therapy \& Allied Technologies. 2010;19(2):83-93.

aatli B, Yildirim N, Olgan S, Koyuncuoglu M, Emekci O, Sayglli U. The role of endometrial thickness for detecting endometrial pathologies in asymptomatic postmenopausal women. Australian and New Zealand Journal of Obstetrics and Gynaecology. 2014;54(1):36-40.

Wong AW, Lao TH, Cheung C, Yeung S, Fan H, Ng P, et al. Reappraisal of endometrial thickness for the detection of endometrial cancer in postmenopausal bleeding: a retrospective cohort study. BJOG: An International Journal of Obstetrics \& Gynaecology. 2016;123(3):439-46.

Basbug A, Dogan O, Yassa M, Pulatoglu C, Kaya AE, Caliskan E. Relationship between adnexal mass and endometrial thickness in postmenopausal period. Medicine. 2018;7(4):848-51.

Piróg M, Kacalska-Janssen O, Bereza T, Jach R. The thin red line-postmenopausal abnormal uterine bleeding with endometrial thickness less than $4 \mathrm{~mm}$. Contemporary Oncology. 2019;23(1):43.

Palipana D, Fomin I, Russell E, Chan B, Wong C, Chan E, et al. Investigating women with postmenopausal bleeding: The utility of endometrial thickness in transvaginal ultrasound. Australian and New Zealand Journal of Obstetrics and Gynaecology. 2020. 